\title{
RESIN PHASE MASS TRANSFER IN ION EXCHANGE BETWEEN DIFFERENT IONS ACCOMPANIED BY RESIN VOLUME CHANGE
}

\author{
TAKESHI KATAOKA AND HIROYUKI YOSHIDA* \\ Department of Chemical Engineering, \\ University of Osaka Prefecture, Sakai, 591
}

\begin{abstract}
Ion exchange between different ions in resin phase was analyzed by applying a model in which the changes of resin diameter and ion diffusivity with the progress of ion exchange were considered simultaneously in addition to the effect of an electric field. Numerical results were applied to systems which exhibit larger and smaller volume changes, respectively. The present model may be used for the case when a large resin volume change occurs, while the simple Nernst-Planck model is applicable to the case of a small-volume change system.
\end{abstract}

\section{Introduction}

The mechanism of mass transfer in the resin phase is one of the important problems of ion exchange operation, and has been studied by many investigators.

The mass transfer in the resin phase involves various complicated factors, such as the effect of the electric field caused by the difference in diffusivity, the volume change of resin particles with the progress of ion exchange, and the activity coefficient of the ionic species in addition to the characteristic factors for the diffusion in a solid. Helfferich et al. ${ }^{2,3,8)}$ proposed the Nernst-Planck model for the resin-phase mass transfer in ion exchange between different ions by considering simply the effect of the electric field. Their theory is based on the following assumptions:

1) Resin phase constructed by a matrix structure is homogeneous. 2) The resin diameter, the diffusivity and the activity coefficient of the ionic species are constant.

However, after the theory was proposed it was reported that there are various systems in which experimental results don't agree with theoretical prediction.

Recently, modifications of the theory of Helfferich et $a l^{2,3,8)}$ have been attempted. Morigh et al. ${ }^{7}$ and Lutze et $a l^{6)}$ presented the theoretical equation by considering the change of the intraparticle diffusivity during ion exchange reaction, and Brooke et al. ${ }^{1)}$ proposed a model taking into account the effect of the

Received February 17, 1975.

Presented at the 11th Kyoto Symposium of The Soc. of Chem. Engrs., Japan, at Kyoto, 1973. activity coefficient of the counter ion into the NernstPlanck model. These theoretical values, unexpectedly, don't agree with experimental results.

The authors ${ }^{5)}$ have reported that the resin volume changes appreciably with the change of gram-equivalent weight of the ion in the resin phase. Therefore, in the ion exchange reaction between ionic species with different gram-equivalent weight, it may be thought that the effect of the change of resin diameter on the ion exchange rate cannot be neglected. In this work, the Nernst-Planck model is modified by considering the changes of resin diameter and the diffusivity of counter ion with the progress of ion exchange. Further, the theoretical equation was compared with the experimental results for systems in which the resin diameter changes slightly ( $\mathrm{R}-\mathrm{Na}-\mathrm{Zn}$ and $\mathrm{R}-\mathrm{Na}-\mathrm{H}$ ) and for systems in which the diameter changes appreciably (R-Ba-Na and $\mathrm{R}-\mathrm{Ba}-\mathrm{H})$.

\section{Theory}

If the resin diameter changes with the progress of ion exchange, the following considerations are required to analyse resin-phase mass transfer.

1) The change of the diffusion length accompanied by the moving boundary.

2) The change of the diffusivity resulting from the volume change of the resin.

3) The movement of the fixed ionic groups caused by the resin volume change.

4) The effect of the activity coefficient gradient.

The item 2) listed above is very interesting and important, but it is difficult to make clear. Accordingly, authors' estimation equation $\mathrm{s}^{5}$ ) for the resin phase self-diffusivity of $Z$ valence ion, that is, Eq. (1) 
for any ion except $\mathrm{Ba}^{2+}$ ion and Eq. (2) for $\mathrm{Ba}^{2+}$ ion, were adopted as an approach to the problem.

$$
\begin{aligned}
\frac{\bar{D}}{D_{L}^{0}} & =0.55 \exp \left(-0.174 Z \frac{V_{H}^{W}}{V} X\right) \\
\left.\frac{\bar{D}}{D_{L}^{0}}\right|_{B a^{2+}} & =0.36 \exp \left(-0.348 \frac{V_{H}^{W}}{V} X\right)
\end{aligned}
$$

When the resin diameter changes with the progress of ion exchange, item 3) will occur. Namely, the ionic group in a position of the resin phase moves with the change of resin diameter, because it is crosslinked by divinyl benzene. This movement causes a change of total concentration per unit volume of the resin. Further, the effect of the electric field is produced by the difference in moving rate of counter ions and fixed ionic groups.

Item 4) listed above is disregarded because nothing is clear for the problem.

Ion exchange between $B^{Z}{ }$ ion in the liquid phase and $A^{Z_{A}}$ ion in the resin phase is expressed by the equation

$$
Z_{B} R-A^{Z_{A}}+Z_{A} B^{Z_{B}} \longrightarrow Z_{B} A^{Z_{A}}+Z_{A} R-B^{Z_{B}}
$$

and the flux of ionic species $i$ is given by the following Nernst-Planck equation:

$$
J_{i}=-\bar{D}_{i}\left(\frac{\partial C_{i}}{\partial r}+\frac{Z_{i} C_{i} \mathscr{F}}{\mathscr{R} T} \frac{\partial \varphi}{\partial r}\right) \quad(i=A, B)
$$

Characteristic conditions of ion exchange, namely, no net current flow and electroneutrality are described as follows.

$$
\begin{aligned}
Z_{A} J_{A}+Z_{B} J_{B} & =v_{r} C_{Y} \\
Z_{A} C_{A}+Z_{B} C_{B} & =C_{Y}
\end{aligned}
$$

where $Y$ is the ionic group fixed by the matrix structure in the resin phase, and $v_{r}$, the moving rate of $Y$ ion to the radial direction, is given by Eq. (7), if a resin is homogeneously expanding or shrinking.

$$
v_{r}=\frac{\mathrm{d} R(t)}{\mathrm{d} t} \frac{r}{R(t)}
$$

Assuming that the change of $C_{Y}$ caused by the volume change is a function of time $t$ only and is independent of $r$, Eqs. (4)-(6) can be combined.

$$
\begin{aligned}
J_{A}= & -\bar{D}_{A} \bar{D}_{B} \frac{Z_{A}^{2} C_{A}+Z_{B}^{2} C_{B}}{Z_{A}^{2} \bar{D}_{A} C_{A}+Z_{B}^{2} \bar{D}_{B} C_{B}} \frac{\partial C_{A}}{\partial r} \\
& +\frac{\bar{D}_{A} Z_{A} C_{A} C_{Y} v_{r}}{Z_{A}^{2} \bar{D}_{A} C_{A}+Z_{B}^{2} \bar{D}_{B} C_{B}}
\end{aligned}
$$

Combination of Eq. (8) with the equation of continuity gives, for a spherical geometry,

$$
\begin{aligned}
\frac{\partial C_{A}}{\partial t}= & \frac{1}{r^{2}} \frac{\partial}{\partial r}\left(r^{2} \bar{D}_{A} \bar{D}_{B} \frac{Z_{A}^{2} C_{A}+Z_{B}^{2} C_{B}}{Z_{A}^{2} \bar{D}_{A} C_{A}+Z_{B}^{2} \bar{D}_{B} C_{B}} \frac{\partial C_{A}}{\partial r}\right. \\
& \left.-r^{2} \frac{\bar{D}_{A} Z_{A} C_{A} C_{Y} v_{r}}{Z_{A}^{2} \bar{D}_{A} C_{A}+Z_{B}^{2} \bar{D}_{B} C_{B}}\right)
\end{aligned}
$$

where the second term of the right-hand side of Eq.
(9) shows the effect of resin diameter change, and if $v_{r}=0$, the equation is reduced to the Nernst-Planck model (hereafter called N. P. model) in the case where the resin diameter is constant.

Next, using dimensionless variables (refer to Nomenclature), Eq. (9) is normalized to Eq. (10).

$$
\begin{aligned}
\frac{\partial U_{A}}{\partial \tau}= & \frac{1}{\rho^{2}} \frac{\partial}{\partial \rho}\left[\rho ^ { 2 } \left(\frac{b U_{A}+U_{Y}}{a U_{A}+U_{Y}} \frac{\partial U_{A}}{\partial \rho}\right.\right. \\
& \left.\left.-\frac{U_{A} U_{Y} V_{r}}{a U_{A}+U_{Y}}\right)\right] \frac{\bar{D}_{A}}{\bar{D}_{A}^{0}}
\end{aligned}
$$

where $\bar{D}_{A}^{0}$ denotes the resin-phase self-diffusivity of A ion, that is, $\bar{D}_{A}$ at $t=0$. In addition, fractional attainment $F_{B}$ is given by Eq. (11).

$$
F_{B}=\frac{4 \pi \int_{0}^{R(t)} C_{B} r^{2} \mathrm{~d} r}{(4 / 3) \pi R_{0}^{3} C_{Y_{0}}}=3 \int_{0}^{\rho_{0}(\tau)} U_{B} \rho^{2} \mathrm{~d} \rho
$$

Initial and boundary conditions are given as follows.

$$
\begin{array}{ll}
\text { (I. C.) } & U_{A}=1, \quad \tau=0 \\
\text { (B. C.) } & U_{A}=0, \quad \rho=\rho_{0}(\tau)=R(t) / R_{0} \\
& \partial U_{A} / \partial \rho=0, \quad \rho=0
\end{array}
$$

Transforming Eq. (10) to the finite difference equation, the following equations are obtained.

$$
\begin{aligned}
& U_{A}(\tau+\Delta \tau, \rho)=\frac{1}{M}\left[G_{P} D_{P} U_{A}(\tau, \rho+\Delta \rho)\right. \\
&+\left(M-G_{P} D_{P}-G_{M} D_{M}\right) U_{A}(\tau, \rho) \\
&+\left.G_{M} D_{M} U_{A}(\tau, \rho-\Delta \rho)\right]-\frac{Z_{A}}{Z_{B}} \alpha \frac{\rho}{\Delta \rho} U_{Y}(\tau) \\
& \quad \times {\left[G_{P P} \frac{U_{A}(\tau, \rho+\Delta \rho)+U_{A}(\tau, \rho)}{2 U_{Y}(\tau)+a\left\{U_{A}(\tau, \rho+\Delta \rho)+U_{A}(\tau, \rho)\right\}}\right.} \\
&-\left.G_{M M} \frac{U_{A}(\tau, \rho)+U_{A}(\tau, \rho-\Delta \rho)}{2 U_{Y}(\tau)+a\left\{U_{A}(\tau, \rho)+U_{A}(\tau, \rho-\Delta \rho)\right\}}\right] \\
& \times\left(1-\frac{\rho_{0}(\tau-\Delta \tau)}{\rho_{0}(\tau)}\right) \\
& G_{P}=\left(1+\frac{\Delta \rho}{2 \rho}\right)^{2}, G_{P P}=\left(1+\frac{\Delta \rho}{2 \rho}\right)^{3}, G_{M}=\left(1-\frac{\Delta \rho}{2 \rho}\right)^{2} \\
& G_{M M}=\left(1-\frac{\Delta \rho}{2 \rho}\right)^{3}, M=\frac{(\Delta \rho)^{2} \bar{D}_{A}^{0}}{\Delta \tau \bar{D}_{A}} \\
& D_{P}=-\frac{2 U_{Y}(\tau)+b\left\{U_{A}(\tau, \rho+\Delta \rho)+U_{A}(\tau, \rho)\right\}}{2 U_{Y}(\tau)+a\left\{U_{A}(\tau, \rho+\Delta \rho)+U_{A}(\tau, \rho)\right\}} \\
& D_{M}= \frac{2 U_{Y}(\tau)+b\left\{U_{A}(\tau, \rho)+U_{A}(\tau, \rho-\Delta \rho)\right\}}{2 U_{Y}(\tau)+a\left\{U_{A}(\tau, \rho)+U_{A}(\tau, \rho-\Delta \rho)\right\}}
\end{aligned}
$$

Stability conditions are given as follows. (Refer to Appendix.)

(A) In the case of $b \leq 0$ and $\alpha \geq 1$

$$
M=2.5
$$

(B) In the case of $b>0$ and $\alpha<1$

$$
M=2.5 \frac{U_{Y}(\tau)+b U_{A}(\tau, 0)}{U_{Y}(\tau)+a U_{A}(\tau, 0)}
$$

As mentioned above, the resin-phase diffusivities were calculated from Eq. (1) or (2). The values of $\rho_{0}(\tau+\Delta \tau)$ 

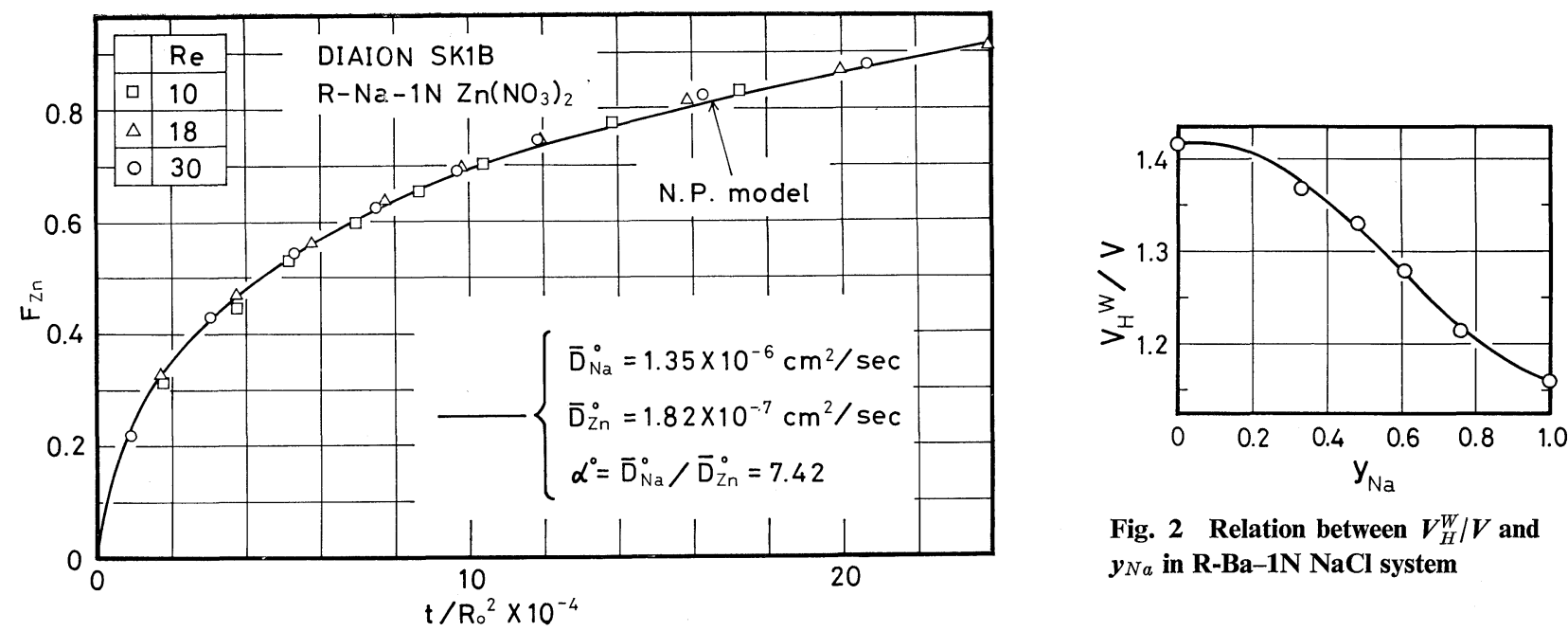

Fig. 2 Relation between $V_{H}^{W} / V$ and $\boldsymbol{y}_{N a}$ in R-Ba-1N NaCl system

Fig. 1 Ion exchange rate in $\mathrm{R}-\mathrm{Na}-1 \mathrm{~N} \mathrm{Zn}\left(\mathrm{NO}_{3}\right)_{2}$ system

was evaluated by substituting $F_{B}(\tau+\Delta \tau)$, which was calculated from Eq. (11), to the relation between $R(\tau)$ and $F_{B}(\tau)$ obtained from the experiment. The radial increment $\Delta \rho$ was $1 / 160$ in the initial state of exchange, and the value was increased with the progress of exchange. This result was the same as that in the case of $1 / 640$ and $1 / 1280$.

\section{Experimental Method}

The ion exchange rates were measured by the single particle method ${ }^{4,5)}$. Table 1 shows the systems and conditions used in the study. The resin particles were observed under a microscope to measure their diameters in water before each experimental run and in the feed solution after each run, respectively.

Since it is difficult to measure simultaneously both the ion exchange rate and the fractional volume change $V_{H}^{W} / V$, the relation between the resin-phase concentration of counter ion and $V_{H}^{W} / V$ were obtained as follows. After the equilibrium resin-phase concentration with the total exchanging ionic concentration of $1 \mathrm{~N}$ solution was measured by column method, several particles were taken from the resin bed and their diameters in solution were measured. Then these particles were contacted with $1 \mathrm{~N} \mathrm{HCl}$ until they changed to $\mathrm{H}$-type perfectly. After washing with distilled water, their diameters were measured again. From these data, the values of $V$ and $V_{H}^{W}$ were calculated, respectively.

\section{Experimental Results and Discussion}

\section{1 Systems in which the resin diameter changes only slightly}

Fig. 1 shows the experimental results of DIAION SK1B R- ${ }^{22} \mathrm{Na}-1 \mathrm{~N} \mathrm{Zn}\left(\mathrm{NO}_{3}\right)_{2}$ system in comparison with calculated results from the N. P. model using the self-diffusivity of $\mathrm{Na}^{+}$and $\mathrm{Zn}^{2+}$, respectively, meas-

\begin{tabular}{lll}
\hline $\begin{array}{l}\text { Table } 1 \\
\text { work } \\
\text { Resin }\end{array}$ & \multicolumn{1}{c}{ Resin, systems and experimental condition in this } \\
\hline DIAION & \multicolumn{1}{c}{ System } & Temperature \\
SK1B & R-22 Na-1N Zn $\left(\mathrm{NO}_{3}\right)_{2}$ & \\
$(X=8)$ & R-22 Na-1N HCl & $25^{\circ} \mathrm{C}$ \\
& R-133 $\mathrm{Ba}-1 \mathrm{~N} \mathrm{NaCl}$ & \\
\hline
\end{tabular}

ured by isotopic ion exchange ${ }^{5)}$. Good agreement between experimental results and theoretical values suggests that the effect of diameter change with the progress of ion exchange may be neglected in this system. In fact, the measured values of $V_{H}^{W} / V$ of $\mathrm{R}-\mathrm{Na}$ and $\mathrm{R}-\mathrm{Zn}$ in $1 \mathrm{~N}$ solution were 1.16 and 1.10 , respectively. This means that the N.P. model may be applied to systems in which resin diameter change is small.

\section{2 System in which the resin diameter changes appreciably}

For this case, $\mathrm{R}-{ }^{133} \mathrm{Ba}-1 \mathrm{~N} \mathrm{NaCl}$ system was selected. Fig. 2 shows the relation between the fractional volume change $V_{H}^{W} / V$ and fractional equivalent concentration in the resin phase $y_{N a}$. The solid line indicates Eq. (19) determined by the least square method using the data in this figure.

$$
\frac{V_{H}^{W}}{V}=1.414+0.1302 y_{N a}-0.8878 y_{N a}^{2}+0.5040 y_{N a}^{3}
$$

The resin diameter change caused by the change of $y_{N a}$ is shown in Fig. 3.

Further, Fig. 4 indicates the change of resin-phase diffusivity of $\mathrm{Na}^{+}$and $\mathrm{Ba}^{2+}$ calculated from Eqs. (1) and (2) with $y_{N a}$. This figure shows that the diffusivities of $\mathrm{Ba}^{2+}$ and $\mathrm{Na}^{+}$ion increase gradually with increasing $y_{N a}$. It is noted that the diffusivity change of $\mathrm{Ba}^{2+}$ ion, a divalent ion, is affected by $V_{H}^{W} / V$ at a rate twice that for $\mathrm{Na}^{+}$ion, a monovalent 


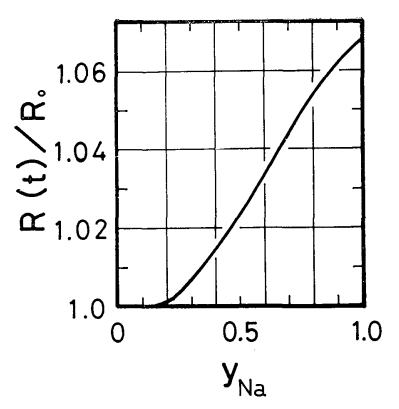

Fig. 3 Relation between $R(t) / R_{0}$ and $y_{N a}$ in R-Ba-1N NaCl system

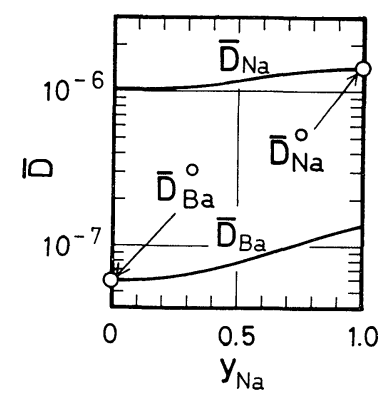

Fig. 4 The change of the diffusivity in $\mathrm{R}-\mathrm{Ba}-\mathbf{1 N} \mathrm{NaCl}$ system

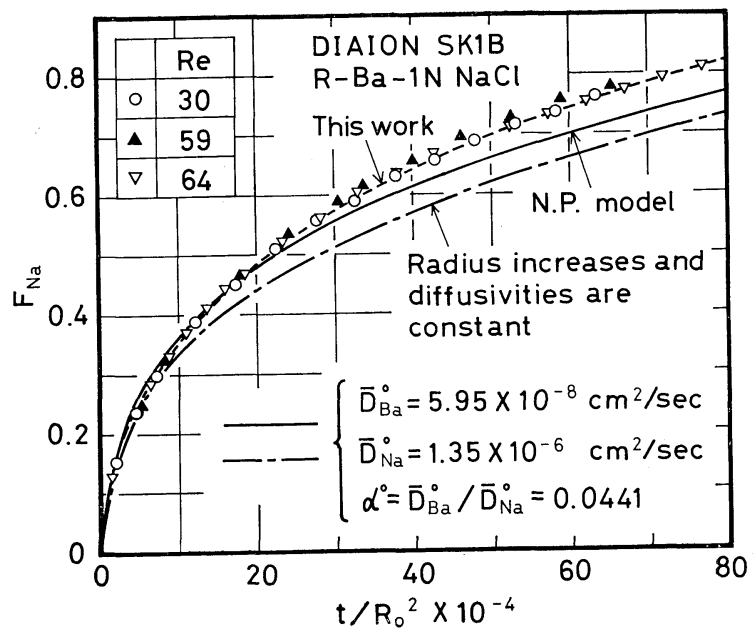

Fig. 5 Exchange rate in R-Ba-1N NaCl system

ion, as is obvious from Eqs. (1) and (2).

The experimental results for $\mathrm{Ba}^{2+}$ type DIAION SK1B-NaCl system are plotted in Fig. 5. The solid line shows numerical results calculated from the N. P. model using the self-diffusivity of $\mathrm{Ba}^{2+}$ and $\mathrm{Na}^{+}$ $i^{5}{ }^{5}$. The one point dashed line shows the results calculated by considering only the effect of the volume change caused by ion exchange based on the present theory, and disregarding the change of diffusivity. This indicates that the rate should be slower than that predicted by the N. P. model. This is because the length of diffusion becomes greater with increase of diameter caused by the progress of ion exchange.

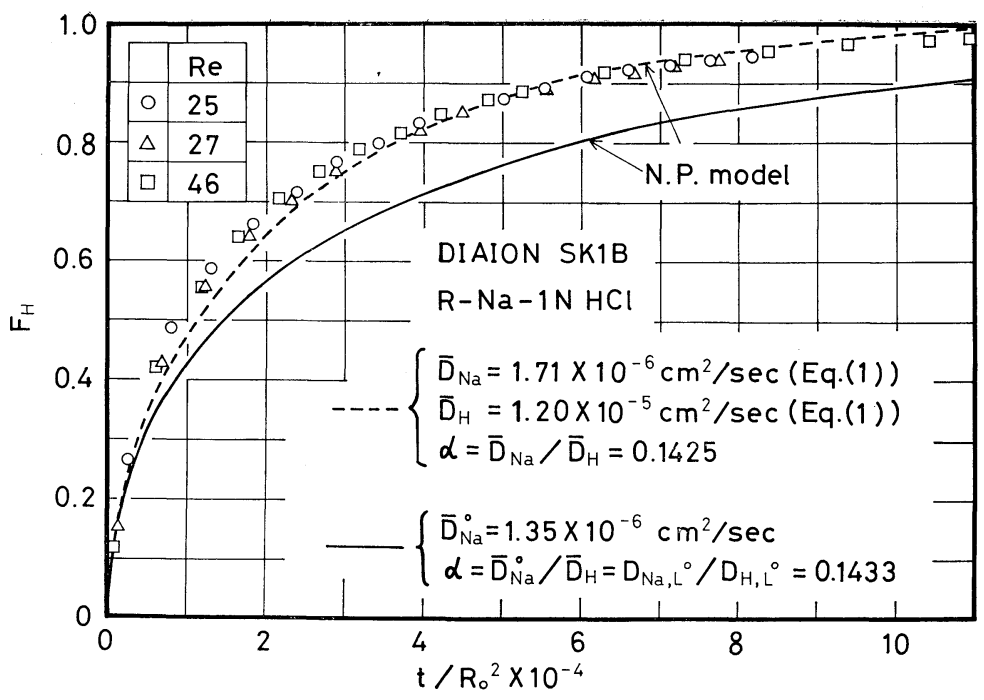

Fig. 6 Exchange rate in $\mathrm{R}-\mathrm{Na}-1 \mathrm{~N} \mathrm{HCl}$ system

Both lines in the figure above-mentioned deviate from the experimental results. The dotted line means the theoretical line calculated by considering both the effect of volume and diffusivity change simultaneously. The calculated result expressing the fractional attainment of $\mathrm{Na}^{+}, F_{N a}$, versus $t / R_{0}^{2}$ agrees fairly well with the experimental result. Therefore, it may be concluded that the ion exchange rate becomes faster than that expected from the N.P. model and that this is because the effect of the increase of diffusivities of $\mathrm{Ba}^{2+}$ and $\mathrm{Na}^{+}$ion with the progress of ion exchange is greater than the negative effect of the increase in diffusion length.

\section{3 R-Na-HCl and R-Ba-HCl system}

Since it is difficult to measure the diffusivity of $\mathrm{H}^{+}$ ion, the ion exchange between $\mathrm{H}^{+}$ion and any other ion has been predicted by a rough assumption, such as that the diffusivity ratio in the resin phase is approximately equal to that in infinitely diluted aqueous solution. In this work, the exchange rate for R$\mathrm{Na}-\mathrm{HCl}$ system with a small diameter change and $\mathrm{R}-\mathrm{Ba}-\mathrm{HCl}$ system with appreciable diameter change were measured and compared with the N. P. model and the present theory.

Fig. 6 shows the experimental results of DIAION SK1B R-Na-1N $\mathrm{HCl}$ system. In this figure, the solid line indicates the theoretical result predicted from the N. P. model by the use of the diffusivity ratio for infinitely diluted aqueous solution and the self-diffusivity of $\mathrm{Na}^{+}$ion ${ }^{5)}$. The dotted line is the theoretical line based on the N. P. model, and with the diffusivity of $\mathrm{Na}^{+}$and $\mathrm{H}^{+}$ion calculated from Eq. (1) by the use of the value of $V_{H}^{W} / V$ for the $H$ type resin in $1 \mathrm{~N}$ solution. The latter result coincides fairly well with the experimental ones.

Next, the relation between $V_{H}^{W} / V$ and $y_{H}$ in the R$\mathrm{Ba}-1 \mathrm{H} \mathrm{HCl}$ system in which the diameter changes 
appreciably is shown in Fig. 7. The solid line shows Eq. (20) obtained from the experiment.

$$
\frac{V_{H}^{W}}{V}=1.417-0.02546 y_{H}-1.050 y_{H}^{2}+0.7145 y_{H}^{3}
$$

Fig. 8 indicates the experimental results of the exchange rate. The dotted line is the theoretical result based on the present model using the Eqs. (1) and (2) for $\mathrm{H}^{+}$and $\mathrm{Ba}^{2+}$ ions, respectively. It is clear that the present model gives reasonable results in this case, too. The one point dashed line shows the theoretical values calculated from the N.P. model using the self-diffusivity of $\mathrm{Ba}^{2+}$ ion and the diffusivity ratio in infinitely diluted aqueous solution. Also, the solid line shows the results obtained from the N. P. model employing the self-diffusivity of $\mathrm{Ba}^{2+}$ ion and the value calculated from Eq. (1) using the experimental value of $V_{H}^{W} / V$ of H-type resin in $1 \mathrm{~N}$ solution. The one point dashed and solid lines deviate from the experimental results appreciably.

\section{Conclusion}

The exchange rate in systems with a slight diameter change and with appreciable diameter change, respectively, were measured, and the results were compared with the N. P. model and the model proposed here considering the changes in resin particle diameter and in diffusivities. The following conclusions were obtained. 1) In systems which exhibit a smaller change in diameter, the N.P. model is applicable. 2) In systems which show an appreciable diameter change, the model proposed in this paper agrees fairly well with the experimental values. 3) The equation to predict resin-phase diffusivity obtained by isotopic ion exchange is applicable for ion exchange between different ions, and the diffusivity of $\mathrm{H}^{+}$ion can also be estimated from Eq. (1). This model should be tested by other systems if the fractional volume change measured at the equilibrium condition cannot express fully the values at unsteady state in resin-phase ion transfer.

\section{Appendix}

To get the numerical solution of Eq. (15), each part of the first term of right side in Eq. (15) should be greater than or equal to zero. Accordingly, the following equation is obtained.

$$
M-G_{P} D_{P}-G_{M} D_{M} \geq 0
$$

If $M$ satisfying Eq. (A-2) is selected, Eq. (15) is stable.

$M \geq M_{\text {max }}=\left(G_{P} D_{P}+G_{M} D_{M}\right)_{\max } \geq\left(G_{P} D_{P}+G_{M} D_{M}\right) \quad(\mathrm{A}-2)$ where $M_{\text {max }}$ means the maximum value of $\left(G_{P} D_{P}+G_{M} D_{M}\right)$. In this calculation, $M=M_{\max }$ was adopted.

\section{Nomenclature}

$$
\begin{aligned}
& a \quad=\left(Z_{A} / Z_{B}\right) \alpha-1 \\
& \text { b } \quad=\left(Z_{A} / Z_{B}\right)-1 \\
& C \quad=\text { concentration of counter ion in }
\end{aligned}
$$

\begin{tabular}{|c|c|c|}
\hline$C_{Y}$ & $\begin{array}{l}=\text { concentration of fixed ionic group } \\
\text { in resin phase }\end{array}$ & [meq. $\left./ \mathrm{cm}^{3}\right]$ \\
\hline$C_{Y_{0}}$ & $=C_{Y}$ at $t=0$ & [meq. $\left./ \mathrm{cm}^{3}\right]$ \\
\hline $\bar{D}$ & $\begin{array}{l}=\text { diffusivity of counter ion in } \\
\text { resin phase }\end{array}$ & {$\left[\mathrm{cm}^{2} / \mathrm{se}\right.$} \\
\hline $\bar{D}^{0}$ & $\begin{aligned}= & \text { self-diffusivity of counter ion in } \\
& \text { resin phase }\end{aligned}$ & {$\left[\mathrm{cm}^{2} / \mathrm{sec}\right.$} \\
\hline$D_{L}^{0}$ & $\begin{array}{l}=\text { diffusivity in infinitely diluted aqueo } \\
\text { solution }\end{array}$ & {$\left[\mathrm{cm}^{2} / \mathrm{se}\right.$} \\
\hline$F$ & $=$ fractional attainment of ion exchang & e $\quad[-$ \\
\hline $\mathscr{F}$ & $=$ Faraday constant & \\
\hline$J$ & $=$ flux of ion & $\mathrm{le} / \mathrm{cm}^{2} \cdot \mathrm{sec}$ \\
\hline$M$ & $=(\Delta \rho)^{2} \bar{D}_{A}^{0} /\left(\Delta \tau \bar{D}_{A}\right)$ & {$[-$} \\
\hline$R_{0}$ & $=$ radius of resin at $t=0$ & {$[\mathrm{cr}$} \\
\hline$R(t)$ & $=$ radius of resin at $t=t$ & {$[\mathrm{cn}$} \\
\hline $\mathscr{R}$ & $=$ gas constant & \\
\hline$r$ & $=$ radial coordinate & [ch \\
\hline$t$ & $=$ time & [se \\
\hline$U_{A}$ & $=Z_{A} C_{A} / C_{Y_{0}}$ & {$[-$} \\
\hline$U_{B}$ & $=Z_{B} C_{B} / C_{Y_{0}}$ & {$[-$} \\
\hline$U_{Y}$ & $=C_{Y} / C_{Y_{0}}$ & {$[-$} \\
\hline$V$ & $=$ volume of resin & {$\left[\mathrm{cm}^{3} / \mathrm{meq}\right.$} \\
\hline$V_{H}^{W}$ & $=$ volume of $\mathrm{H}$-type resin in water & {$\left[\mathrm{cm}^{3} / \mathrm{meq}\right.$} \\
\hline$V_{r}$ & $\begin{aligned}= & \left(Z_{A} / Z_{B}\right)\left(R_{0} v_{r} / \bar{D}_{B}\right) \text { dimensionless } \mathrm{m} \\
& \text { rate of fixed ionic group in radial dir }\end{aligned}$ & \\
\hline
\end{tabular}

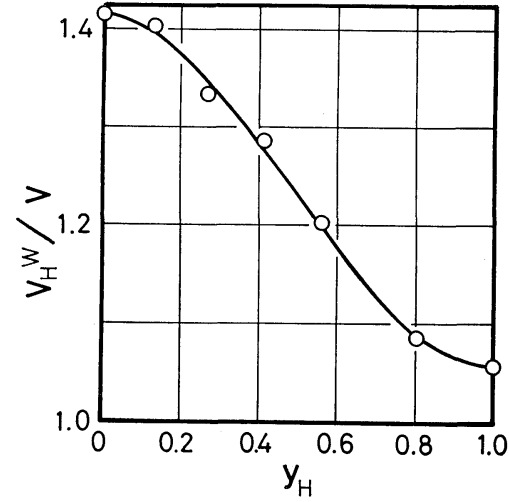

Fig. 7 Relation between $V_{H}^{W} / V$ and $y_{H}$ in R-Ba-1N $\mathbf{H C l}$ system

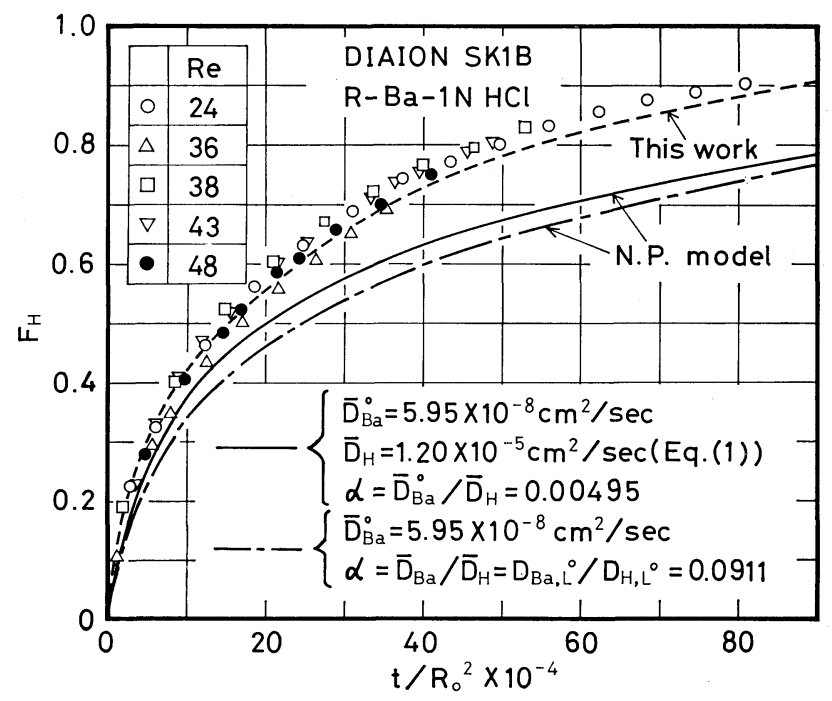

Fig. 8 Ion exchange rate in $\mathrm{R}-\mathrm{Ba}-1 \mathrm{~N} \mathrm{HCl}$ system 


\begin{tabular}{|c|c|c|}
\hline$v_{r}$ & $\begin{array}{l}=\text { moving rate of fixed ionic group in } \\
\text { radial direction }\end{array}$ & $\mathrm{m} / \mathrm{sec}]$ \\
\hline$X$ & $=$ degree of crosslinking & {$[\%]$} \\
\hline$y$ & $=$ fractional equivalent of ion in resin phase & {$[-]$} \\
\hline$Z$ & $=$ ionic valence & {$[-]$} \\
\hline$\alpha$ & $=\bar{D}_{A} / \bar{D}_{B}$ diffusivity ratio & {$[-]$} \\
\hline$\alpha^{0}$ & $=\bar{D}_{A}^{0} / \bar{D}_{B}^{0}$ self-diffusivity ratio & {$[-]$} \\
\hline$\rho$ & $=r / R_{0}$ & {$[-]$} \\
\hline$\rho_{0}$ & $=R(t) / R_{0}$ & {$[-]$} \\
\hline$\tau$ & $=\bar{D}_{A}^{0} t / R_{0}^{2}$ & {$[-]$} \\
\hline & $=$ electric potential & \\
\hline
\end{tabular}

\section{Literature Cited}

1) Brooke, N. M.: Trans. Faraday Soc., 64, 3383 (1968).

2) Helfferich, F.: J. Chem. Phys., 35, 39 (1963).

3) Helfferich, F. and M. S. Plessert: ibid., 28, 418 (1958).

4) Katakoka, T., M. Sato, T. Nishiki and K. Ueyama: Kagaku Kögaku, 33, 873 (1969).

5) Kataoka, T., H. Yoshida and H. Sanada: J. Chem. Eng. Japan, 7, 105 (1974).

6) Lutze, W. and N. Miekely: J. Phys. Chem., 75, 2483 (1971).

7) Morich, C. R. and G. Rao: Chem. Eng. Sci., 20, 889 (1965).

8) Plessert, M. S. and F. Helfferich: J. Chem. Phys., 29, 1064 (1958).

\title{
MASS TRANSFER TO A ROTATING SPHERE IN AN AXIAL STREAM
}

\author{
TAKESHI FURUTA*, TAKAO JIMBO, MORIO OKAZAKI \\ AND RYOzo TOEI \\ Department of Chemical Engineering, Kyoto University, \\ Kyoto, 606
}

\begin{abstract}
The average and local mass transfer coefficients for a rotating sphere in an axial stream were measured by an electrochemical method over a range of $\operatorname{Re}_{r}$ from $10^{3}$ to $5 \times 10^{4}$ and four kinds of $R_{t}$. Fifteen isolated electrodes were used to obtain the local mass transfer rate. The convective diffusion equation for laminar boundary layer on the sphere was solved by the Lighthill transformation on the basis of numerically calculated shear stress distribution by the series expansion method. The numerical results, using the measured mainstream velocity, were in good agreement with the experimental ones over the laminar boundary layer region except very near the separation point.
\end{abstract}

\section{Introduction}

Studies of heat or mass transfer to a rotating sphere in an axial stream are not only important to determine the transport process between particles and fluids in a particle-fluid mixed flow, but also are of purely hydrodynamic interest.

Recently, Noordsij and Rotte ${ }^{8)}$ measured the average mass transfer coefficients for a rotating sphere placed in a stream with its axis of rotation perpendicular to the stream. Later Tanaka and Tago $^{10)}$ performed experiments on average mass transfer from a rotating camphor sphere, in which the air stream flowed parallel and perpendicular to the axis of rotation. There are, however, no experiments on the local mass transfer coefficients.

Received June 4, 1975.

Presented at Tokushima Meeting of The Soc. of Chem. Engrs., Japan, at Tokushima, July, 1975.
On the other hand, there have been a few theoretical studies of this problem. Using Kármán's momentum integral equation of motion, Schlichting ${ }^{9}$ calculated the velocity profiles and other dynamic characteristics for a sphere rotating in an axial stream where the mainstream velocity $U$ was assumed to be the same as that in an inviscid fluid stream. Hoskin ${ }^{3)}$ also analyzed its laminar boundary layer equation under the same conditions as Schlichting ${ }^{9}$ by means of the series expansion method. However, they did not discuss any heat or mass transfer process. Siekmann $^{10)}$ solved numerically its laminar thermal boundary layer equation for $P r=1.0$ and 0.7 , utilizing the results of Hoskin ${ }^{3}$. In practical conditions, however, the pressure distribution around a sphere in a stream is different from that of an inviscid fluid, so that Siekmann's results may not be applied directly.

The purposes of the present work are to measure the local mass transfer coefficients for a rotating 